\title{
Antifungal prophylaxis of patients undergoing allogenetic hematopoietic stem cell transplantation in China: a multicenter prospective observational study
}

Lei Gao ${ }^{1}$, Yuqian Sun², Fanyi Meng ${ }^{3}$, Mingzhe Han ${ }^{4}$, He Huang $^{5}$, Depei Wu' ${ }^{6}$ Li Yư ${ }^{7}$, Hanyun Ren ${ }^{8}$, Xiaojun Huang ${ }^{2+}$ and Xi Zhang ${ }^{1 *+}$

\begin{abstract}
Background: Antifungal prophylaxis is currently regarded as the gold standard in situations with allo-genetic hematopoietic stem cell transplantation (allo-HSCT). However, the epidemiological information regarding prophylaxis of invasive fungal diseases (IFDs) is not clear in China.

Methods: We report the first large-scale (1053 patients) observational study of the prophylaxis and management of IFDs among patients with allo-HSCT in China.

Results: The incidence rates of IFD after primary antifungal prophylaxis (PAP), secondary antifungal prophylaxis (SAP), and non-prophylaxis were 22.7 vs. 38.6 vs. $68.6 \%$, respectively $(P=0.0000)$. The median time from transplantation to IFD was 45 days in PAP patients, 18 days in SAP patients, and 12 days in non-prophylaxis patients. Aspergillus spp. represents the most common type of fungal infection. Independent risk factors for IFD in allo-HSCT patients with PAP were age, having human leukocyte antigen (HLA)-haploidentical or matched unrelated donor, decreased albumin levels, and the use of itraconazole as the prophylactic antifungal agent. Among SAP transplant recipients, there was no significant risk factor for IFDs. The incidence rates of overall survival (OS) in the PAP, SAP, and no prophylaxis groups were $85.07,78.80$, and 74.82 , respectively $(P=0.01)$.
\end{abstract}

Conclusions: This observational study indicates that prophylaxis of IFD is helpful to reduce the incidence of IFDs and improve the OS of patients after allo-HSCT.

Keywords: Invasive fungal diseases, Allogenetic, Stem cell, Transplantation, Prophylaxis, China

\section{Key points}

This first large-scale observational study of invasive fungal disease (IFD) in China indicated that prophylaxis of IFD is helpful to improve the OS of patients after allogenetic hematopoietic stem cell transplantation (alloHSCT). For patients with a history of IFD, effective prevention of IFD is particularly important because they have higher incidence of IFD.

\footnotetext{
* Correspondence: zhangxxi@sina.com

${ }^{\dagger}$ Equal contributors

'Xinqiao Hospital, Third Military Medical University, Chongqing 400037, China Full list of author information is available at the end of the article
}

\section{Background}

Although the control of bacterial infections in patients with hematological malignancies has been significantly improved with broad-spectrum antibiotics in the past decades, treating invasive fungal diseases (IFDs) is still a major problem in these patients, especially in patients undergoing allogenetic hematopoietic stem cell transplantation (allo-HSCT) [1-3]. Epidemiological data from the USA, China, and parts of Europe have shown that the incidence of IFDs in patients after allo-HSCT has increased dramatically in the past several years, substantially increasing the morbidity and mortality rates [4-9].

The diagnosis and treatment guidelines for IFDs, including some that are specific to HSCT patients, have 
been developed by academic societies in different world regions [10-12]. Notwithstanding the guidance and new forms of antifungal agents, treatment is often delayed because of nonspecific disease presentation and a lack of reliable diagnostic techniques, leading to poor clinical outcomes. Prophylaxis of IFDs is currently regarded as the gold standard in situations with allo-HSCT. Over the years, different scientific societies have established a series of recommendations for antifungal prophylaxis based on prospective studies performed with different drugs [13-17].

However, until recently, data on the prophylaxis of IFDs and real-world management of IFDs in patients with allo-HSCT have been gleaned primarily from single-center and retrospective studies in China. Here, we report the first large-scale observational study of the prophylaxis and management of IFDs among adults and children who have undergone allo-HSCT in China. Data came from the China Assessment of Antifungal Therapy in Hematological Disease (CAESAR) study.

\section{Methods}

Study design

The CAESAR study was a multicenter, prospective, observational study performed in 35 hematology centers across China, including two children's hospitals. Subjects were consecutive patients of any age with a hematological malignancy who were hospitalized during the study period either after allogeneic or autologous HSCT or to receive intravenous chemotherapy. The overall study methods have been described previously $[7,18]$. This observational study was conducted as a part of the CAESAR study and focused on the prophylaxis of IFDs in 1053 patients who underwent allo-HSCT in 31 HSCT centers.

All patients in each study center who were hospitalized and had undergone allo-HSCT between January 1, 2011 and October 30, 2011 were enrolled in this study. Data were collected by chart reviews and included the following parameters: demographic characteristics, antecedent hematologic disease, type of HSCT, conditioning regimen, IFD risk factors, clinical features suggestive of IFD, laboratory findings such as imaging, histopathology, and fungal cultures, treatment outcome of IFD, and mortality. Each IFD was categorized as proven, probable, or possible, according to the European Organization for Research and Treatment of Cancer/Invasive Fungal Infections Cooperative Group and the National Institute of Allergy and Infectious Diseases Mycoses Study Group (EORTC/MSG) 2008 criteria [19]. Patients were diagnosed as having suspected IFD if they had IFD risk factors; were observed to have symptoms, radiological abnormalities, or indirect microbiological evidence of fungal infection; and were treated empirically with antifungal agents but could not be diagnosed with proven, probable, or possible IFD according to the EORTC/MSG
2008 criteria [20]. Patients were followed for 6 months after the date of transplantation; the follow-up was completed on April 30, 2012. In all, 18 patients were lost to follow-up.

\section{Statistical analysis}

In accordance with common practice and to maximize diagnostic accuracy, the incidence of IFD was mainly calculated based on proven and probable cases combined. The cumulative incidence was calculated as the incidence of proven plus probable IFD for the first 187 days after transplantation, divided by the number of cases at risk. Data were grouped according to primary antifungal prophylaxis (PAP) or secondary antifungal prophylaxis (SAP). PAP refers to patients without a history of fungal infection and need to be protected to avoid fungal infection after HSCT [21]. SAP is a rational strategy for patients with proven or probable IFD within 6 months before transplantation [22]. In particular, all allo-HSCT patients $(n=1045)$ in the CAESAR study were included in the analysis of overall survival (OS) for comparison of the OS differences in the PAP, SAP, and no prophylaxis groups. The characteristics of all of the alloHSCT patients were described in the CAESAR study [7].

Statistics were primarily descriptive and were compared using analysis of variance, the Wilcoxon rank-sum test, or the chi-squared test, as appropriate. Risk factors for IFD were analyzed using univariate analysis followed by multivariate logistic regression. Kaplan-Meier analysis and the log-rank test were used to compare OS between different groups of patients. A two-sided $P$ value of less than 0.05 was denoted as statistically significant. All statistical analyses were performed with SAS software version 9.1 (SAS, Cary, NC, USA).

\section{Results}

\section{Patient characteristics and antifungal prophylaxis} regimen

A total of 1053 patients who underwent allo-HSCT were enrolled from 31 HSCT centers across China. Their baseline demographic and clinical characteristics at the time of admission for transplantation are shown in Table 1. Among them, 906 patients received prophylactic antifungal treatment before or after transplantation. Previous IFDs were noted in 88 patients $(8.4 \%, 88 / 1053)$, including 7 with proven IFDs and 81 with probable IFDs.

The average duration of antifungal prophylaxis before HSCT was $10.9 \pm 6.56$ days $(10.8 \pm 6.57$ days in the PAP group and $12.0 \pm 6.43$ days in the SAP group). Single antifungal agents were the most common prophylaxis regimen $(667 / 818$ in the PAP group and $69 / 88$ in the SAP group). Fluconazole $(429 / 818,64.9 \%)$ and voriconazole $(32 / 88,46.4 \%)$ were the most widely used agents for PAP and SAP, respectively (Table 1). All antifungal 
Table 1 Patient characteristics and antifungal prophylaxis regimen

\begin{tabular}{|c|c|c|c|}
\hline Characteristic & $\operatorname{PAP}(N=818)$ & $\operatorname{SAP}(N=88)$ & Non-antifungal prophylaxis $(n=147)$ \\
\hline Age, mean (range/\%)/case & $28.5(1-63)$ & $32.22-58)$ & $29.6(4-61)$ \\
\hline $0-6$ & $33(4 \%)$ & $3(3.4 \%)$ & $4(2.7 \%)$ \\
\hline$>6-16$ & $129(15.8 \%)$ & $10(11.4 \%)$ & $20(13.6 \%)$ \\
\hline$>16-40$ & $488(59.7 \%)$ & 49 (55.7\%) & $85(57.8 \%)$ \\
\hline$>40-65$ & $168(20.5 \%)$ & $26(29.5 \%)$ & $38(25.9 \%)$ \\
\hline \multicolumn{4}{|l|}{ Gender } \\
\hline Male & $505(61.7 \%)$ & $51(58.0 \%)$ & 87 (59.2 \%) \\
\hline Female & $313(38.3 \%)$ & $37(42.0 \%)$ & 60 (40.8\%) \\
\hline \multicolumn{4}{|l|}{ E-COG } \\
\hline 0 & $227(27.8 \%)$ & $18(20.5 \%)$ & $48(32.7 \%)$ \\
\hline 1 & $438(53.5 \%)$ & $54(61.4 \%)$ & $74(50.3 \%)$ \\
\hline 2 & 89 (10.9\%) & $11(12.5 \%)$ & $17(11.6 \%)$ \\
\hline 3 & $51(6.2 \%)$ & $5(5.7 \%)$ & $7(4.8 \%)$ \\
\hline 4 & $13(1.6 \%)$ & - & $1(0.7 \%)$ \\
\hline \multicolumn{4}{|l|}{ Underlying disease } \\
\hline Acute myeloid leukemia & $289(35.3 \%)$ & $44(50.0 \%)$ & $43(29.3 \%)$ \\
\hline Acute lymphocytic leukemia & $241(29.5 \%)$ & $32(36.4 \%)$ & 37 (25.2 \%) \\
\hline Chronic myeloid leukemia & $100(12.2 \%)$ & $3(3.4 \%)$ & $23(15.6 \%)$ \\
\hline Aplastic anemia & $69(8.4 \%)$ & - & $13(8.8 \%)$ \\
\hline Myelodysplastic syndrome & $62(7.6 \%)$ & $2(2.3 \%)$ & $16(10.9 \%)$ \\
\hline Non-Hodgkin's lymphoma & $23(2.8 \%)$ & $4(4.5 \%)$ & $4(2.7 \%)$ \\
\hline Chronic lymphocytic leukemia & $4(0.5 \%)$ & - & - \\
\hline Multiple myeloma & $2(0.2 \%)$ & - & $1(0.7 \%)$ \\
\hline Hodgkin's disease & $2(0.2 \%)$ & - & $3(2.0 \%)$ \\
\hline Myeloprolififerative neoplasms & $2(0.2 \%)$ & - & - \\
\hline Solid tumor & $1(0.1 \%)$ & - & - \\
\hline Hereditary and metabolic disorders & $9(1.1 \%)$ & - & - \\
\hline Paroxysmal nocturnal hemoglobinuria & $1(0.1 \%)$ & - & $1(0.7 \%)$ \\
\hline Others $^{a}$ & $13(1.6 \%)$ & $3(3.4 \%)$ & $6(4.1 \%)$ \\
\hline \multicolumn{4}{|l|}{ Transplantation type } \\
\hline HLA-matched related (sibling) & $335(41.0 \%)$ & 35 (39.8\%) & 77 (52.4 \%) \\
\hline Haploidentical & $269(32.9 \%)$ & $26(29.5 \%)$ & $35(23.8 \%)$ \\
\hline Unrelated & $213(26.0 \%)$ & 27 (30.7 \%) & 35 (23.8 \%) \\
\hline \multicolumn{4}{|l|}{ Source of stem cells } \\
\hline PB & $417(51.0 \%)$ & 67 (76.1\%) & $105(71.4 \%)$ \\
\hline $\mathrm{BM}+\mathrm{PB}$ & $348(42.5 \%)$ & $19(21.6 \%)$ & $20(13.6 \%)$ \\
\hline BM & $33(4.0 \%)$ & - & 7 (4.8 \%) \\
\hline$C B$ & $15(1.8 \%)$ & 1 (1.1\%) & $3(2.0 \%)$ \\
\hline $\mathrm{BM}+\mathrm{CB}$ & $3(0.4 \%)$ & - & - \\
\hline$B M+P B+C B$ & $2(0.2 \%)$ & 1 (1.1\%) & $8(5.4 \%)$ \\
\hline \multicolumn{4}{|l|}{ Conditioning regimen } \\
\hline \multicolumn{4}{|l|}{ Myeloablative } \\
\hline Yes & $729(89.1 \%)$ & $83(94.3 \%)$ & $124(84.4 \%)$ \\
\hline No & 89 (10.9\%) & 5 (5.7 \%) & $23(15.6 \%)$ \\
\hline
\end{tabular}


Table 1 Patient characteristics and antifungal prophylaxis regimen (Continued)

\begin{tabular}{|c|c|c|c|}
\hline \multicolumn{4}{|l|}{ Total body radiotherapy } \\
\hline Yes & $122(14.9 \%)$ & $23(26.1 \%)$ & $13(8.8 \%)$ \\
\hline No & $696(85.1 \%)$ & $65(73.9 \%)$ & $134(91.2 \%)$ \\
\hline \multicolumn{4}{|l|}{ Antithymocyte globulin } \\
\hline Yes & $516(63.1 \%)$ & $52(59.1 \%)$ & $64(43.5 \%)$ \\
\hline No & $302(36.9 \%)$ & $36(40.9 \%)$ & $83(56.5 \%)$ \\
\hline Concomitant disease & $137(16.7 \%)$ & $22(25.0 \%)$ & $23(15.6 \%)$ \\
\hline Cardiovascular disease & $24(2.9 \%)$ & $2(2.3 \%)$ & $4(2.7 \%)$ \\
\hline Diabetes & $24(2.9 \%)$ & $2(2.3 \%)$ & $6(4.1 \%)$ \\
\hline Viral hepatitis & $22(2.7 \%)$ & $9(10.2 \%)$ & $4(2.7 \%)$ \\
\hline CMV infection & 1 (0.1\%) & $2(2.3 \%)$ & 1 (0.7 \%) \\
\hline Tuberculosis & $14(1.7 \%)$ & $3(3.4 \%)$ & $2(1.4 \%)$ \\
\hline Autoimmune diseases & $9(1.1 \%)$ & $1(1.1 \%)$ & - \\
\hline Others $^{\mathrm{b}}$ & $61(7.5 \%)$ & $5(5.7 \%)$ & $8(5.4 \%)$ \\
\hline \multicolumn{4}{|l|}{$\mathrm{aGVHD}$} \\
\hline I-II aGVHD & $257(31.4 \%)$ & $27(30.7 \%)$ & $40(27.2 \%)$ \\
\hline III-IV aGVHD & $57(7.0 \%)$ & 7 (8.0 \%) & $15(10.2 \%)$ \\
\hline \multicolumn{4}{|l|}{ cGVHD } \\
\hline Limited & $57(7.0 \%)$ & $9(10.3 \%)$ & $10(6.85)$ \\
\hline Extensive & $24(2.9 \%)$ & $2(2.3 \%)$ & $4(2.7 \%)$ \\
\hline \multicolumn{4}{|l|}{ Drugs for IFD prophylaxis } \\
\hline Single drug & $667(81.5 \%)$ & $69(78.4 \%)$ & - \\
\hline Fluconazole & $429(64.3 \%)$ & $8(11.6 \%)$ & - \\
\hline Itraconazole & $103(15.4 \%)$ & $17(24.6 \%)$ & - \\
\hline Voriconazole & $71(10.6 \%)$ & $32(46.4 \%)$ & - \\
\hline Caspofungin & $4(0.6 \%)$ & $6(8.7 \%)$ & - \\
\hline Amphotericin B & $2(0.3 \%)$ & $1(1.4 \%)$ & - \\
\hline Drug combination & $151(18.5 \%)$ & $19(21.6 \%)$ & - \\
\hline \multicolumn{4}{|l|}{ Route of administration } \\
\hline Oral & $466(57.0 \%)$ & $29(33.0 \%)$ & - \\
\hline Intravenous & $214(26.2 \%)$ & $37(42.0 \%)$ & - \\
\hline Oral + intravenous & $138(16.9 \%)$ & $22(25.0 \%)$ & - \\
\hline Time of prophylaxis before HSCT mean (SD), days & $10.8(6.57)$ & $12.0(6.43)$ & - \\
\hline
\end{tabular}

E-COG Eastern Cooperative Oncology Group, $P A P$ primary antifungal prophylaxis, $S A P$ secondary antifungal prophylaxis, $B M$ bone marrow, $P B$ peripheral blood, $C B$ cord blood, $C M V$ cytomegalovirus, $a G V H D$ acute graft versus host disease, $C G V H D$ chronic graft versus host disease, IFD invasive fungal diseases, $H S C T$ hematopoietic stem cell transplantation

${ }^{a}$ Others include chronic myelomonocytic leukemia $(n=3)$, T lymphoblastic lymphoma leukemia $(n=2)$, mixed-lineage leukemia $(n=10)$, aggressive NK cell leukemia $(n=3)$, myeloid sarcoma $(n=1)$, plasmablasticlymphoma $(n=3)$

${ }^{b}$ Others include infectious disease $(n=18)$, digestive system diseases $(n=10)$, cholecystolithiasis $(n=7)$, mouth disease $(n=8)$, urological diseases $(n=6)$, intestinal diseases $(n=7)$, endocrine diseases $(n=7)$, surgery $(n=5)$, cerebrovascular disease $(n=4)$, tumor $(n=2)$

agents were given in accordance with the recommended doses and schedule.

\section{Efficacy of PAP and SAP on the prophylaxis of IFD occurrence}

In the 1053 patients, 321 (30.5\%) patients were identified as having an IFD, including $13(1.2 \%)$ with proven IFD, 81 (7.7 \%) with probable IFD, and $227(21.6 \%)$ with possible IFD. The incidence rates of proven, probable, and possible IFD in the PAP, SAP, and non-prophylaxis groups were 0.7 vs. 0.0 vs. $4.8 \%, 7.0$ vs. 11.4 vs. $9.5 \%$, and 15.0 vs. 27.3 vs. $54.4 \%$, respectively (Table 2). The median time from transplantation to IFD was 45 days in the PAP group (interquartile range (IQR), 16 to 75), 18 days in the SAP group (IQR, 7 to 26), and 12 days in the non-prophylaxis group (IQR, 0 to 93), respectively. 
Table 2 The recurrence rates of IFD in patients from the PAP, SAP, and non-prophylaxis groups

\begin{tabular}{llll}
\hline Characteristic & PAP $(N=818)$ & SAP $(N=88)$ & Non-prophylaxis $(n=147)$ \\
\hline IFD patients & $186(22.7 \%)$ & $34(38.6 \%)$ & $101(68.7 \%)$ \\
Proven & $6(0.7 \%)$ & $0(0.0 \%)$ & $7(4.8 \%)$ \\
Probable & $57(7.0 \%)$ & $10(11.4 \%)$ & $14(9.5 \%)$ \\
Possible & $123(15.0 \%)$ & $24(27.3 \%)$ & $80(54.4 \%)$ \\
Patients without IFD & $632(77.3 \%)$ & $54(61.4 \%)$ & $46(31.3 \%)$ \\
\hline
\end{tabular}

\section{Characteristics of patients with IFD occurrence}

Thirteen patients were diagnosed with proven IFD (6 in the PAP group and 7 in the non-prophylaxis group), and 81 patients were diagnosed with probable IFD (57 in the PAP group, 10 in the SAP group, and 14 in the nonprophylaxis group). Among the 94 patients with IFD, 56 were male and 38 were female. The underlying diseases were acute myeloid leukemia $(n=32)$, acute lymphocytic leukemia $(n=32)$, myelodysplastic syndrome $(n=10)$, chronic myeloid leukemia $(n=8)$, non-Hodgkin's lymphoma $(n=5)$, aplastic anemia $(n=5)$, and others $(n=2)$. Twenty patients received human leukocyte antigen (HLA)-matched sibling HSCT, 42 patients received HLA-haploidentical HSCT, and 32 patients received HLA-matched unrelated HSCT.

Detailed data regarding the distribution of fungal pathogens and the methods of microbiological diagnoses of
IFDs are shown in Table 3. Of the 89 etiological pathogens identified in patients with proven or probable IFDs, 62 (69.7\%) were Aspergillus, including 19 that were exclusively diagnosed with two positive galactomannan tests. Unspecified Aspergillus was the most common mold (31 cases), followed by Aspergillus flavus (5 cases), and Aspergillus fumigatus (2 case) isolates.

The infection sites of all categories of IFD were also analyzed. Of all the 54 infection sites identified in patients with IFDs, the most common location of infection was the lower respiratory tract $(64.8 \%, 35 / 54)$, followed by blood stream infections only (13.0\%, 7/ $54)$, central nervous system (5.6 \%, 3/54), spleen (1.9\%, 1/54), and three other sites (14.8\%, 8/54). Further analysis showed that Aspergillus is the main pathogen of lower respiratory tract fungal infection $(85.7 \%, 30 / 35)$. In other infection sites, there was no

Table 3 Identified etiological pathogens in IFD cases

\begin{tabular}{|c|c|c|c|c|}
\hline Fungal species & Overall population & PAP & SAP & Non-prophylaxis \\
\hline Overall & 89 & 55 & 7 & 27 \\
\hline Candida spp. & 27 & 14 & 3 & 10 \\
\hline Unclassified Candida & 8 & 5 & 0 & 3 \\
\hline Candida tropicalis & 3 & 2 & 0 & 1 \\
\hline Candida albicans & 3 & 2 & 0 & 1 \\
\hline Candida parapsilosis & 3 & 2 & 0 & 1 \\
\hline Candida krusei & 3 & 1 & 1 & 1 \\
\hline Candida glabrata & 1 & 1 & 0 & 0 \\
\hline Candida albicans & 5 & 1 & 2 & 2 \\
\hline Not specified ${ }^{a}$ & 1 & 0 & 0 & 1 \\
\hline Aspergillus spp. & 62 & 41 & 4 & 17 \\
\hline Positive GM tests only & 19 & 15 & 1 & 3 \\
\hline Aspergillus flavus & 5 & 3 & 0 & 2 \\
\hline Aspergillus versicolor & 1 & 1 & 0 & 0 \\
\hline Aspergillus fumigatus & 2 & 2 & 0 & 0 \\
\hline Aspergillus albicans & 1 & 0 & 0 & 1 \\
\hline Mucor & 1 & 0 & 0 & 1 \\
\hline Cryptococcus & 1 & 1 & 0 & 0 \\
\hline Pityrosporion ovale & 1 & 1 & 0 & 0 \\
\hline Not specified ${ }^{b}$ & 31 & 18 & 3 & 10 \\
\hline
\end{tabular}

ancluding cases with yeasts identified in tissue but negative cultures

$\mathrm{b}^{\mathrm{b}}$ Including cases with hyphae identified in tissue but negative cultures 
Table 4 Risk factors for proven/probable IFD among allo-HSCT patients in the PAP group

\begin{tabular}{|c|c|c|c|c|c|c|}
\hline \multirow[t]{2}{*}{ Factor } & \multicolumn{3}{|l|}{ Univariate analysis } & \multicolumn{3}{|c|}{ Multivariate analysis } \\
\hline & $\begin{array}{l}\text { Patients with proven/ } \\
\text { probable IFD }(n / N)\end{array}$ & $\begin{array}{l}\text { Incidence of proven/ } \\
\text { probable IFD (\%) }\end{array}$ & $\begin{array}{l}\text { Comparison } \\
(P \text { value })\end{array}$ & SE & $\begin{array}{l}\text { OR } \\
(95 \% \mathrm{Cl})\end{array}$ & $\begin{array}{l}\text { Comparison } \\
\text { ( } P \text { value) }\end{array}$ \\
\hline \multicolumn{7}{|l|}{ Age } \\
\hline$\leq 18$ years old & $9 / 182$ & 4.95 & 0.15 & 0.44 & $2.78(1.18-6.55)$ & 0.02 \\
\hline$>18$ years old & $54 / 636$ & 8.49 & & & & \\
\hline \multicolumn{7}{|l|}{ Transplantation type } \\
\hline HLA-matched related (sibling) & $10 / 335$ & 2.99 & 0.0001 & & & \\
\hline Haploidentical & $31 / 269$ & 11.52 & & 0.65 & $6.08(1.69-21.86)$ & 0.01 \\
\hline Unrelated & $22 / 213$ & 10.33 & & 0.60 & $8.07(2.50-26.10)$ & 0.00 \\
\hline \multicolumn{7}{|l|}{ Antithymocyte globulin } \\
\hline Yes & $49 / 516$ & 4.64 & 0.01 & 0.57 & $0.40(0.13-1.24)$ & 0.11 \\
\hline No & $14 / 302$ & 9.50 & & & & \\
\hline \multicolumn{7}{|l|}{ Glucocorticoids $^{\mathrm{a}}$} \\
\hline Yes & $58 / 678$ & 8.55 & 0.05 & 0.58 & $1.37(0.44-4.23)$ & 0.58 \\
\hline No & $5 / 140$ & 3.57 & & & & \\
\hline \multicolumn{7}{|l|}{$G V H D^{b}$} \\
\hline Non-GVHD & $31 / 428$ & 7.24 & 0.15 & 0.40 & $1.63(0.74-3.58)$ & 0.22 \\
\hline aGVHD I-II & $16 / 230$ & 6.96 & & & & \\
\hline aGVHD III-IV & $8 / 52$ & 15.38 & & & & \\
\hline cGVHD local & $2 / 60$ & 3.33 & & & & \\
\hline cGVHD extensive & $3 / 27$ & 11.11 & & & & \\
\hline \multicolumn{7}{|l|}{ Prolonged, profound neutropenia } \\
\hline ANC $>500 / \mathrm{mm}^{3}$ & $1 / 14$ & 7.14 & 0.0006 & & & \\
\hline ANC $<500 / \mathrm{mm}^{3},<10$ days & $6 / 142$ & 4.23 & & 1.22 & $0.40(0.04-4.33)$ & 0.45 \\
\hline ANC $<500 / \mathrm{mm}^{3}, 10-14$ days & $4 / 176$ & 2.27 & & 1.28 & $0.14(0.01-1.73)$ & 0.13 \\
\hline ANC $<500 / \mathrm{mm}^{3},>14$ days & $52 / 486$ & 10.70 & & 1.16 & $0.99(0.10-9.60)$ & 0.99 \\
\hline \multicolumn{7}{|l|}{ EBV viremia $^{c}$} \\
\hline Yes & $10 / 62$ & 16.13 & 0.03 & 0.44 & $1.35(0.58-3.17)$ & 0.49 \\
\hline No & $43 / 646$ & 6.66 & & & & \\
\hline Untested & $10 / 110$ & 9.09 & & & & \\
\hline \multicolumn{7}{|l|}{ CMV viremia $^{c}$} \\
\hline Yes & $33 / 267$ & 12.36 & 0.004 & 0.33 & $1.18(0.61-2.26)$ & 0.63 \\
\hline No & $29 / 528$ & 5.49 & & & & \\
\hline Untested & $1 / 23$ & 4.35 & & & & \\
\hline \multicolumn{7}{|l|}{ Renal impairment } \\
\hline Yes & $10 / 60$ & 16.67 & 0.019 & 0.43 & $1.38(0.59-3.24)$ & 0.46 \\
\hline No & $53 / 758$ & 6.99 & & & & \\
\hline \multicolumn{7}{|l|}{ Decreased albumin } \\
\hline Yes & $39 / 317$ & 12.30 & 0.0001 & 0.31 & $1.98(1.08-3.62)$ & 0.03 \\
\hline No & $24 / 501$ & 4.79 & & & & \\
\hline \multicolumn{7}{|l|}{ Time of IFD prophylaxis } \\
\hline$<35$ days & $33 / 396$ & 8.33 & 0.60 & 0.30 & $1.33(0.74-2.39)$ & 0.35 \\
\hline$\geq 35$ days & $30 / 416$ & 7.21 & & & & \\
\hline
\end{tabular}


Table 4 Risk factors for proven/probable IFD among allo-HSCT patients in the PAP group (Continued)

\begin{tabular}{|c|c|c|c|c|c|c|}
\hline \\
\hline \multicolumn{7}{|l|}{$\begin{array}{l}\text { Urugs for IFU prophylaxis } \\
\text { Fluconazole/fluconazole + itraconazole }\end{array}$} \\
\hline Itraconazole $e^{e}$ & $14 / 103$ & 13.59 & & 0.53 & $3.14(1.11-8.86)$ & 0.03 \\
\hline Voriconazole & $2 / 71$ & 2.82 & & 0.85 & $0.95(0.18-5.01)$ & 0.95 \\
\hline Other $^{d}$ & $8 / 144$ & 5.56 & & & & \\
\hline
\end{tabular}

ANC absolute neutrophil count, EBV Epstein-Barr virus

${ }^{a}$ Including dexamethasone, methylprednisolone, prednisone, and hydrocortisone

${ }^{b}$ By multivariate analysis, OR is for CGVHD extensive/aGVHD III and IV degree versus CGVHD local/aGVHD I and II degree/non-GVHD

${ }^{\mathrm{C}} \mathrm{By}$ multivariate analysis, OR is for with EBV viremia versus without EBV viremia or untested/CMV viremia versus without CMV viremia or untested

dIncluding caspofungin, micafungin, amphotericin B, fluconazole + caspofungin, fluconazole + micafungin, fluconazole + voriconazole, itraconazole + micafungin,

voriconazole + caspofungin, and fluconazole + caspofungin + voriconazole

eCompared with others

The italicized data reflected significant difference

significant difference in the infection rate between $A s$ pergillus and Candida.

\section{Risk factors for proven and probable IFD}

The risk factors for proven and probable IFDs among PAP and SAP transplant recipients are presented in
Tables 4 and 5. Univariate analyses revealed that HLAhaploidentical HSCT, HLA-matched unrelated HSCT, the use of antithymocyte globulin, prolonged profound neutropenia ( $>14$ days), renal impairment, decreased albumin levels, and Epstein-Barr virus and cytomegalovirus viremia were independent risk factors for proven

Table 5 Risk factors for proven/probable IFD among allo-HSCT patients in SAP group

\begin{tabular}{|c|c|c|c|c|c|c|}
\hline \multirow[t]{2}{*}{ Factor } & \multicolumn{3}{|l|}{ Univariate analysis } & \multicolumn{3}{|c|}{ Muitivariate analysis } \\
\hline & $\begin{array}{l}\text { Patients with proven/ } \\
\text { probable IFD }(n / N)\end{array}$ & $\begin{array}{l}\text { Incidence of proven/ } \\
\text { probable IFD (\%) }\end{array}$ & Comparison ( $P$ value) & SE & OR $(95 \% \mathrm{Cl})$ & Comparison ( $P$ value) \\
\hline \multicolumn{7}{|l|}{ Transplantation type } \\
\hline HLA-matched related (sibling) & $1 / 35$ & 2.86 & 0.08 & & & \\
\hline Haploidentical & $5 / 26$ & 19.23 & & 1.77 & $2.48(0.08-79.46)$ & 0.61 \\
\hline Unrelated & $4 / 27$ & 14.81 & & 1.77 & $2.24(0.07-72.11)$ & 0.65 \\
\hline \multicolumn{7}{|l|}{ Antithymocyte globulin } \\
\hline Yes & $9 / 52$ & 17.31 & 0.04 & 1.68 & $2.49(0.09-67.66)$ & 0.59 \\
\hline No & $1 / 36$ & 2.78 & & & & \\
\hline \multicolumn{7}{|l|}{ Prolonged, profound neutropenia } \\
\hline ANC $>500 / \mathrm{mm}^{3}$ & $0 / 1$ & 0.00 & 0.23 & 1.13 & $5.90(0.65-53.89)$ & 0.12 \\
\hline ANC $<500 / \mathrm{mm}^{3},<10$ days & $1 / 18$ & 5.56 & & & & \\
\hline ANC $<500 / \mathrm{mm}^{3}, 10-14$ days & $0 / 17$ & 0.00 & & & & \\
\hline ANC $<500 / \mathrm{mm}^{3},>14$ days & $9 / 52$ & 17.31 & & & & \\
\hline \multicolumn{7}{|l|}{ Liver impairment } \\
\hline Yes & $5 / 24$ & 20.83 & 0.12 & 0.74 & $2.07(0.49-8.79)$ & 0.33 \\
\hline No & $5 / 64$ & 7.81 & & & & \\
\hline \multicolumn{7}{|l|}{ Time of IFD prophylaxis } \\
\hline$<35$ days & $5 / 40$ & 12.50 & 1.00 & 0.76 & $1.10(0.25-4.86)$ & 0.90 \\
\hline$\geq 35$ days & $5 / 48$ & 10.42 & & & & \\
\hline \multicolumn{7}{|l|}{ Drugs for IFD prophylaxis } \\
\hline Fluconazole/itraconazole/ & $3 / 27$ & 11.11 & 1.00 & 0.96 & $0.87(0.13-5.64)$ & 0.88 \\
\hline \multicolumn{7}{|l|}{ Fluconazole + itraconazole ${ }^{b}$} \\
\hline Voriconazole $^{b}$ & $4 / 32$ & 12.50 & & 0.91 & $1.50(0.25-8.96)$ & 0.66 \\
\hline Others $^{a}$ & $3 / 29$ & 10.34 & & & & \\
\hline
\end{tabular}

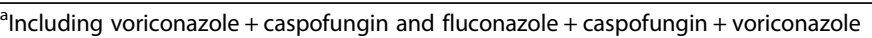

${ }^{b}$ Compared with others

The italicized data reflected significant difference 
and probable IFDs in allo-HSCT patients with PAP $(P<$ 0.05). Among the allo-HSCT patients with SAP, the use of antithymocyte globulin was the only obviously significant risk factor $(P=0.043)$.

Multivariate analyses demonstrated that independent risk factors for proven and probable IFDs in allo-HSCT patients with PAP were age $>18$ years (odds ratio (OR), 2.78; $95 \%$ confidence interval $(\mathrm{CI}), 1.18-6.55 ; P<0.05)$, having an HLA-matched unrelated donor (OR, 8.07; $95 \% \mathrm{CI}, 2.50-26.10 ; \quad P<0 \quad .01)$, having an HLAhaploidentical donor (OR, 6.08; $95 \% \mathrm{CI}, 1.69-21.86$; $P$ $<0.01$ ), decreased albumin levels (OR, 1.98; $95 \% \mathrm{CI}$, $1.08-3.62 ; P<0.05)$, and the use of itraconazole as the antifungal prophylactic agent (OR, 3.41; $95 \% \mathrm{CI}, 1.11-$ 8.86; $P<0$.05) (Table 4). Among the SAP transplant recipients, there was no significant risk factor for proven or probable IFD.

\section{Overall antifungal therapy}

A total of 321 IFD patients were provided therapeutic antifungal treatment. A single antifungal agent was used for treatment in 116 (36.1\%) patients; 144 (44.9\%) patients required two agents, and $61(19.0 \%)$ patients required three or more agents during the course of their treatment. Among the PAP transplant recipients, itraconazole (34.4 \%, 64/186 in all-category IFD; $46.1 \%, 29 / 63$ in proven or probable IFD) was the most popular agent for the initial therapy. Among the SAP and nonprophylaxis transplant recipients, voriconazole $(36.3 \%$, $49 / 135$ in all-category IFD; $25.8 \%, 8 / 31$ in proven or probable IFD) was the most common agent for the initial therapy. Whether the patients in PAP, SAP, or nonprophylaxis groups, voriconazole as a single agent or in combination with other agents $(35.8 \%, 115 / 321)$ was the most popular agent for antifungal treatment.

The median overall treatment duration in patients who started and completed antifungal treatment while hospitalized was 36 days (IQR, 21-65) for PAP transplant recipients, 39 days (IQR, 24-58) for SAP transplant recipients, and 35 days (IQR, 20-67) for nonprophylaxis transplant recipients. A total of 175 patients (54.5\%, 94 in the PAP group, 20 in the SAP group, and 61 in the non-prophylaxis group) continued treatment after leaving the hospital.

\section{Outcomes including mortality}

At the end of follow-up, 171 patients had died, resulting in an overall mortality rate of $16.2 \%(171 / 1053)$. Compared with the overall study population, the mortality rate was markedly higher in patients with proven $(5 / 13$; $38.5 \%)$, probable $(19 / 81 ; 23.5 \%)$, or possible $(58 / 227$; 25.6 \%) IFDs. Furthermore, the mortality rate among patients treated for suspected IFD despite failing to meet the EORTC/MSG diagnostic criteria [16] $(25 / 166 ; 15.1 \%)$ was higher than that among patients who were not suspected of having IFD and, therefore, not treated with antifungal agents $(64 / 566 ; 11.3 \%$ ) (Fig. $1, P<0.001)$. In addition, we compared the incidence of OS in the PAP, SAP, and no prophylaxis groups. There was a significant difference among the three groups (85.07 vs. 78.80 vs. $74.82 \%$, respectively, Fig. $2, P=0.01$ ).

\section{Discussion}

Invasive infections remain major infectious threats to patients undergoing allo-HSCT and are associated with high fatality rates [23-28]. The diagnosis of IFDs continues to be difficult to establish because they do not manifest with specific clinical or radiographic signs or symptoms [29]. In the recent years, there has been a concerted effort to identify alternative procedures for the future diagnosis of fungus. They include the targeting of fungal antigens by enzyme-linked immunosorbent assay (ELISA) or lateral flow devices (LFDs) [30], detection of siderophores [31], and amplification of fungal nucleic acids from tissue and body fluids [32] as well as application of matrix-assisted laser desorption ionization TOF mass spectrometry (MALDI-TOF MS) [33]. Each of the above methods has advantages and disadvantages, and not all of which have entered clinical practice [34]. The difficulty in obtaining a timely diagnosis as well as the high morbidity and mortality rates associated with IFDs provide a rationale for antifungal prophylaxis in patients undergoing allo-HSCT. Nonetheless, antifungal prophylaxis remains a topic of some controversy, with no clear consensus among different centers [35-37].

The CAESAR study is the first population-based and the largest prospective observational study of the incidences of IFDs in patients receiving HSCT [7]. This

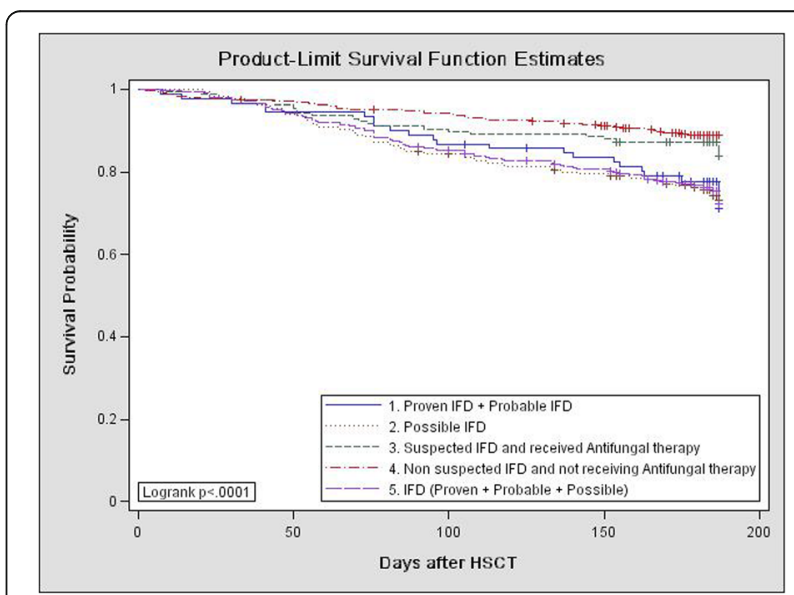

Fig. 1 Overall survival of patients with proven/probable IFD, possible IFD, or suspected IFD and who received antifungal therapy; those with no suspected IFD and who did not receive antifungal therapy; and those without any IFD (proven, probable, or possible) 


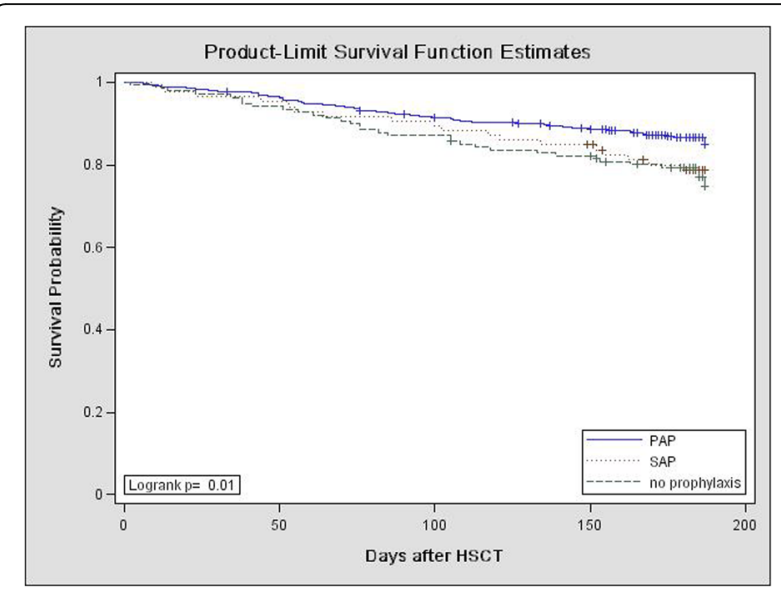

Fig. 2 Overall survival of allo-HSCT patients receiving PAP, SAP, or no prophylaxis

observational study was conducted as a part of the CAESAR study and focused on the prophylaxis of IFDs in patients who underwent allo-HSCT. In the present analysis of adults and children at risk for IFD due to allo-HSCT, non-prophylaxis transplant recipients had a higher incidence of proven/probable/possible IFD than PAP and SAP transplant recipients at 6 months (68.6 vs. 22.7 and $38.6 \%, P=0.0000)$. This result was in accord with the classic randomized clinical trials on PAP in HSCT recipients and patients with hematological malignancies [3840]. Furthermore, the OS was significantly different among PAP, SAP, and no prophylaxis patients. It was revealed that antifungal prophylaxis was helpful to reduce the incidence of IFD and improve the survival of patients after transplantation.

The most recently published European guidelines pointed out that SAP should be administered to patients with a previous IFD to prevent recurrence of the previous IFD or onset of a new IFD during a new at-risk phase, mainly referring to a prolonged neutropenic period induced by chemotherapy or a phase of severe immunosuppression after allo-HSCT [16]. Several studies have reported success rates for SAP, which was proven to be effective in preventing IFD recurrence [41, 42]. In the study, the cumulative incidence of IFD increased particularly rapidly during the first month after transplantation in the SAP group and non-prophylaxis group, suggesting that this is a high-risk period during which health care providers should pay particularly close attention to signs of emerging IFD in SAP and nonprophylaxis patients. On the contrary, the median time of IFD occurrence in the PAP group was 45 days after HSCT. This finding indicated that patients who had a previous IFD and had no antifungal prophylaxis were more likely to experience a breakthrough fungal infection in the early stage after transplantation.
As $22.7 \%$ of patients treated with PAP and $38.6 \%$ of patients treated with SAP in the study went on to develop proven, probable, or possible IFD, there remains an unmet need for education about the appropriate timing and choice of antifungal agent for prophylaxis in China. Among PAP transplant recipients, the most commonly prescribed prophylactic agents were fluconazole and itraconazole, which may be less effective than posaconazole [43]. The use of itraconazole as the antifungal prophylactic agent also proved the independent risk factor for IFD occurrence in our study. An update to the cost-effectiveness of posaconazole vs. fluconazole or itraconazole in the prevention of IFD among neutropenic patients in the USA has shown that posaconazole is a cost-effective alternative to fluconazole or itraconazole in the prevention of IFD among neutropenic patients [44]. Among SAP transplant recipients, there was no significant risk factor for proven or probable IFD. The characteristics of the observational study and the small sample size of the patients treated with SAP may be the main reasons. Prospective and randomized studies assessing the risk factors for SAP are needed in the future.

The epidemiological characteristics of IFD continue to evolve in transplant patients. A major contributor is the widespread use of azole prophylaxis since the early 1990s, which results in less candidiasis but more frequent mold infections in hematologic malignancies [25, 45]. In most [46-48] but not all [49] cases, Aspergillus spp. represent the most frequent cause of fungal-related morbidity in patients with HSCT. In our study, Aspergillus spp. also predominated in culture-proven or histologically proven pathogens and was more than two times as common as Candida spp. The mortality among patients who developed IFD (24.1\%) was almost double than that in the overall population of HSCT patients (15.0\%), and regression analysis confirmed that the development of IFD is a significant independent risk factor for death, highlighting the grave prognosis of those with IFD and the need for a timely diagnosis and prompt treatment. The incidence rates of invasive aspergillosis, however, need to be interpreted with caution as establishing this diagnosis often requires invasive procedures that are difficult to perform in severely ill patients. The low rate of proven/probable IFD in the present study suggests the need to improve diagnostic techniques to treat IFD as early and accurately as possible. Under the existing conditions, in order to reduce the IFDrelated mortality of allo-HSCT patients, preemptive antifungal therapy should be given to patients with possible or suspected IFD.

The current study has several limitations. The main limitation was its observational nature. Confounding factors cannot be controlled effectively in observational studies, frequently due to biased selection of patients or 
treatment protocols. In the analysis of mortality by antifungal therapy, the uncontrolled study design means that no firm conclusions can be drawn regarding the relative effect of different treatments. Another limitation was the relatively short duration. The fact that the overall treatment duration was not recorded in patients who were still taking antifungal treatment after discharge from the hospital further limited data analysis. A longer follow-up would have allowed a more comprehensive analysis of patients who developed IFD after discharge. Procedures for diagnosis, prophylaxis, and treatment of IFD were not prespecified in the protocol but were performed according to usual practice and local clinical guidelines [19]. Furthermore, in some centers, diagnostic procedures were conducted only among patients with suspected clinical signs or symptoms of IFD. The observational study also meant that the diagnosis of specific infections, although guided by EORTC/MSG 2008 criteria, was limited to the data collected according to local hospitals' usual practice; there was no mandatory requirement for diagnostic microbiological testing or the use of a centralized laboratory to validate the results.

\section{Conclusions}

In conclusion, the results of the present observational study indicate that prophylaxis of IFD among patients receiving allo-HSCT for hematological malignancy in China is broadly in line with the recommended practice and is helpful to reduce the incidence of IFD and improve the OS of patients after transplantation. For patients with a history of IFD, active and effective prevention of fungal infections is particularly important because these patients have a higher incidence of IFD occurrence and a shorter incubation period. Different from the traditional view, for the allo-HSCT patients with PAP, itraconazole was a less effective treatment than other antifungal drugs. Aspergillus spp. represents the most common type of fungal infection in patients with allo-HSCT. Due to the limited diagnostic techniques, it is necessary to give antifungal therapy to patients who do not meet the EORTC/MSG 2008 criteria but show clinical evidence of fungal infection.

\footnotetext{
Abbreviations

allo-HSCT: Allo-genetic hematopoietic stem cell transplantation; CAESAR: China Assessment of Antifungal Therapy in Hematological Disease; ELISA: Enzyme-linked immunosorbent assay; EORTC/MSG: European Organization for Research and Treatment of Cancer/Invasive Fungal Infections Cooperative Group and the National Institute of Allergy and Infectious Diseases Mycoses Study Group; HLA: Human leukocyte antigen; IFDs: Invasive fungal diseases; LFDs: Lateral flow devices; MALDI-TOF MS: Matrix-assisted laser desorption ionization TOF mass spectrometry; OS: Overall survival; PAP: Primary antifungal prophylaxis; SAP: Secondary antifungal prophylaxis
}

\section{Acknowledgements}

The authors would like to thank the patients and investigators at each study site, without whom this study would not have been possible. The CAESAR study group investigators not listed as authors were as follows: Jing Chen, Shanghai Children's Medical Center, Shanghai; Zhixiang Shen, Rui Jin Hospital Affiliated to Shanghai Jiao Tong University School of Medicine, Shanghai; Juan Li and Waiyi Zou, The First Affiliated Hospital of Sun Yat-Sen University, Guangzhou; Chun Wang, Jun Yang, and Yu Cai, The First People's Hospital of Shanghai, Shanghai; Yang Xiao and Yonghua Li, The General Hospital of Guangzhou Military Command of PLA, Guangzhou; Yongping Song and Yuewen Fu, Henan Tumor Hospital affiliated to Zhengzhou University, Zhengzhou; Kang Yu, Xudong Hu, and Xingzhou Ren, The First Affiliated Hospital of Wenzhou Medical College, Wenzhou; Huisheng Ai and Jianhui Qiao, Chinese PLA 307 Hospital, Beijing; Jianmin Wang and Xianmin Song, Changhai Hospital of the Second Military Medical University, Shanghai; Hai Bai and Chunbang Wang, The General Hospital of Lanzhou Military Area, Lanzhou; Yongmin Tang and Heping Shen, Children's Hospital of Zhejiang University Medical school, Hangzhou; Jianyong Li and Xiaoyan Zhang, Jiangsu Province Hospital, Nanjing; Xin Du and Chengwei Luo, Guangdong General Hospital, Guangzhou; Jian Ouyang and Yong Xu, Nanjing Drum Tower Hospital, Nanjing; Huo Tan and Runhui Zheng, The First Affiliated Hospital of Guangzhou Medical University, Guangzhou; Ting Liu and Juan Xu, West China Hospital, Sichuan University, Chengdu; Jin Zhou and Liming Li, The First Hospital of Harbin Medical University, Harbin; Jun Ma, Harbin Hematologic Tumor Institution, Harbin; Zhuogang Liu, Shengjing Hospital of China Medical University, Shenyang; Jianda Hu, Fujian Medical University Union Hospital, Fuzhou; Ping Zou and Yong You, Wuhan Union Hospital, Huazhong University of Science and Technology, Wuhan; Lin Liu and Li Wang, The First Affiliated Hospital of Chongqing Medical University, Chongqing; Yan Li and Ran Gao, The First Affiliated Hospital of China Medical University, Shenyang; Zhanxiang Liu, Chinese PLA General Hospital (301 Hospital), Beijing; Mangju Wang, The First Hospital of Peking University, Beijing; Guopan Yu, Nanfang Hospital, Nanfang Medical University, Guangzhou; Ling Wang, Rui Jin Hospital Affiliated to Shanghai Jiao Tong University School of Medicine, Shanghai; Jun Wang, The First Affiliated Hospital of Soochow University, Suzhou; Guixin Zhang, Institute of Hematology and Blood Diseases Hospital, Chinese Academy of Medical Sciences, Tianjin; Xia Qin, Shanghai Children's Medical Center, Shanghai; Liya Ma and Wangzhuo Xie, The First Affiliated Hospital of Medical School of Zhejiang University, Hangzhou, China.

\section{Funding}

This work was supported by Merck Sharp \& Dohme (China), who sponsored the study. The support for the manuscript development was provided by Merck \& Co., Inc. (Whitehouse Station, NJ).

\section{Availability of data and materials}

The datasets supporting the conclusions of this article are included within the article.

\section{Authors' contributions}

$\mathrm{XH}$ and $\mathrm{XZ}$ contributed to the conception and design of the study. FM, MZ, $H H, D W, L Y$, and HR were involved in the development of the methodology and participated in the analysis. LG and YS interpreted the data and drafted the paper. All authors contributed in the writing, review, and revision of the article and final approval for submission.

Consent for publication

Not applicable.

\section{Competing interests}

The authors declare that they have no competing interests.

\section{Ethics approval and consent to participate}

This observational study was conducted in accordance with the Declaration of Helsinki, International Conference on Harmonization Good Clinical Practice, and nationally mandated ethical requirements. The study protocol and informed consent document were reviewed and approved by the ethics committee of Peking University People's Hospital. All participating institutions obtained ethical approval separately before initiation of the study. All study participants provided informed consent.

\section{Author details}

${ }^{1}$ Xinqiao Hospital, Third Military Medical University, Chongqing 400037, China. ${ }^{2}$ Peking University Institute of Hematology, Peking University People's Hospital, Beijing Key Laboratory of Hematopoietic Stem Cell Transplantation, Beijing 100044, China. ${ }^{3}$ Nanfang Hospital, Nanfang Medical University, Guangzhou, China. ${ }^{4}$ Institute of Hematology and Blood Diseases Hospital, 
Chinese Academy of Medical Sciences, Tianjin, China. ${ }^{5}$ The First Affiliated Hospital of Medical School of Zhejiang University, Hangzhou, China. ${ }^{6}$ The First Affiliated Hospital of Soochow University, Suzhou, China. ${ }^{7}$ Chinese PLA General Hospital (301 Hospital), Beijing, China. ${ }^{8}$ The First Hospital of Peking University, Beijing, China

Received: 19 March 2016 Accepted: 24 August 2016

Published online: 23 September 2016

\section{References}

1. Girmenia C, Raiola AM, Piciocchi A, Algarotti A, Stanzani M, Cudillo L, et al. Incidence and outcome of invasive fungal diseases after allogeneic stem cell transplantation: a prospective study of the Gruppo Italiano Trapianto Midollo Osseo (GITMO). Biol Blood Marrow Transplant. 2014;20:872-80.

2. Lv M, Huang XJ. Allogeneic hematopoietic stem cell transplantation in China: where we are and where to go. J Hematol Oncol. 2012;5:10.

3. Lin $R$, Liu Q. Diagnosis and treatment of viral diseases in recipients of allogeneic hematopoietic stem cell transplantation. J Hematol Oncol. 2013;6:94.

4. Kontoyiannis DP, Marr KA, Park BJ, Alexander BD, Anaissie EJ, Walsh TJ, et al. Prospective surveillance for invasive fungal infections in hematopoietic stem cell transplant recipients, 2001-2006: overview of the Transplant-Associated Infection Surveillance Network (TRANSNET) Database. Clin Infect Dis. 2010; 50:1091-100.

5. Neofytos D, Horn D, Anaissie E, Steinbach W, Olyaei A, Fishman J, et al. Epidemiology and outcome of invasive fungal infection in adult hematopoietic stem cell transplant recipients: analysis of Multicenter Prospective Antifungal Therapy (PATH) Alliance registry. Clin Infect Dis. 2009; 48:265-73.

6. Pagano L, Caira M, Nosari A, Van Lint MT, Candoni A, Offidani M, et al. Fungal infections in recipients of hematopoietic stem cell transplants: results of the SEIFEM B-2004 studyeSorveglianza Epidemiologica Infezioni Fungine Nelle Emopatie Maligne. Clin Infect Dis. 2007:45:1161-70.

7. Sun Y, Meng F, Han M, Zhang X, Yu L, Huang H, et al. Epidemiology, management, and outcome of invasive fungal disease in patients undergoing hematopoietic stem cell transplantation in China: a multicenter prospective observational study. Biol Blood Marrow Transplant. 2015;21:1117-26.

8. Lai Y, Chen Y, Hu D, Jiang M, Liu Q, Liu L, et al. Multicenter phase ii study of a combination of cyclosporine a, methotrexate and mycophenolate mofetil for GVHD prophylaxis: results of the Chinese Bone Marrow Transplant Cooperative Group (CBMTCG). J Hematol Oncol. 2014;7:59.

9. Chang $Y$, Zhao X, Xu L, Zhang X, Wang $Y$, Han W, et al. Donor-specific anti-human leukocyte antigen antibodies were associated with primary graft failure after unmanipulated haploidentical blood and marrow transplantation: a prospective study with randomly assigned training and validation sets. J Hematol Oncol. 2015:8:84.

10. Arendrup MC, Dzajic E, Jensen RH, Johansen HK, Kjaeldgaard P, Knudsen JD, et al. Epidemiological changes with potential implication for antifungal prescription recommendations for fungaemia: data from a nationwide fungaemia surveillance programme. Clin Microbiol Infect. 2013;19:E343-53.

11. Mousset S, Buchheidt D, Heinz W, Ruhnke M, Cornely OA, Egerer G, et al. Treatment of invasive fungal infections in cancer patients-updated recommendations of the Infectious Diseases Working Party (AGIHO) of the German Society of Hematology and Oncology (DGHO). Ann Hematol. 2014; 93:13-32.

12. Alothman AF, Al-Musawi T, Al-Abdely HM, Salman JA, Almaslamani M, Yared $\mathrm{N}$, et al. Clinical practice guidelines for the management of invasive Candida infections in adults in the Middle East region: expert panel recommendations. J Infect Public Health. 2014;7:6-19.

13. Pappas PG, Kauffman CA, Andes D, Benjamin Jr DJ, Calandra TF, Edwards Jr $\mathrm{JE}$, et al. Clinical practice guidelines for the management of candidiasis: 2009 update by the Infectious Diseases Society of America. Clin Infect Dis. 2009:48:503-35.

14. Cornely OA, Böhme A, Buchheidt D, Einsele H, Heinz WJ, Karthaus M, et al. Primary prophylaxis of invasive fungal infections in patients with hematologic malignancies. Recommendations of the Infectious Diseases Working Party of the German Society for Haematology and Oncology. Haematologica. 2009;94:113-22.

15. Marr KA, Bow E, Chiller T, Maschmeyer G, Ribaud P, Segal B, et al. Fungal infection prevention after hematopoietic cell transplantation. Bone Marrow Transplant. 2009;44:483-7.
16. Maertens J, Marchetti $\mathrm{O}$, Herbrecht R, Cornely OA, Flückiger U, Frêre $\mathrm{P}$, et al. European guidelines for antifungal management in leucemia and hematopoietic stem cell transplant recipients: summary of the ECIL 3-2009 update. Bone Marrow Transplant. 2011;46:709-18.

17. Baden LR, Bensinger W, Angarone M, Casper C, Dubberke ER, Freifeld AG, et al. Prevention and treatment of cancer-related infections. J Natl Compr Canc Netw. 2012;10:1412-45.

18. Sun Y, Huang $\mathrm{H}$, Chen J, Li J, Ma J, Li J, et al. Invasive fungal infection in patients receiving chemotherapy for hematological malignancy: a multicenter, prospective, observational study in China. Tumor Biol. 2015;36:757-67.

19. De Pauw B, Walsh TJ, Donnelly JP, Stevens DA, Edwards JE, Calandra T, et al. Revised definitions of invasive fungal disease from the European Organization for Research and Treatment of Cancer/Invasive Fungal Infections Cooperative Group and the National Institute of Allergy and Infectious Diseases Mycoses Study Group (EORTC/MSG) Consensus Group. Clin Infect Dis. 2008;46:1813-21.

20. The Chinese Invasive Fungal Infection Committee. The diagnostic criteria and treatment guideline of invasive fungal infection in patients with Hematological disease/malignances (3rd version). Zhonghua Nei Ke Za Zhi [Chinese Journal of Internal Medicine]. 2010;49:451-4 [Article in Chinese].

21. Girmenia C, Barosi G, Piciocchi A, Arcese W, Aversa F, Bacigalupo A, et al. Primary prophylaxis of invasive fungal diseases in allogeneic stem cell transplantation: revised recommendations from a consensus process by Gruppo Italiano Trapianto Midollo Osseo (GITMO). Biol Blood Marrow Transplant. 2014;20:1080-8.

22. Allinson $\mathrm{K}$, Kolve H, Gumbinger HG, Vormoor HJ, Ehlert K, Groll AH. Secondary antifungal prophylaxis in paediatric allogeneic haematopoietic stem cell recipients. J Antimicrob Chemother. 2008;61:734-42.

23. Huang XJ. Current status of haploidentical stem cell transplantation for leukemia. J Hematol Oncol. 2008;1:27.

24. Wan L, Zhang Y, Lai Y, Jiang M, Song Y, Zhou J, et al. Effect of granulocytemacrophage colony-stimulating factor on prevention and treatment of invasive fungal disease in recipients of allogeneic stem-cell transplantation: a prospective multicenter randomized phase IV trial. J Clin Oncol. 2015. [Epub ahead of print].

25. Montesinos P, Rodríguez-Veiga R, Boluda B, Martínez-Cuadrón D, Cano I, Lancharro $A$, et al. Incidence and risk factors of post-engraftment invasive fungal disease in adult allogeneic hematopoietic stem cell transplant recipients receiving oral azoles prophylaxis. Bone Marrow Transplant. 2015;50:1465-72.

26. Wang $C H$, Kan $L P$, Lin HA, Chang FY, Wang NC, Lin TY, et al. Clinical efficacy and safety of primary antifungal prophylaxis with posaconazole versus fluconazole in allogeneic blood hematopoietic stem cell transplantation recipients - a retrospective analysis of a single medical center in Taiwan. Microbiol Immunol Infect. 2014. doi:10.1016/j.jmii.2014.07.009 [Epub ahead of print].

27. Girmenia C, Ferretti A, Barberi W. Epidemiology and risk factors for invasive fungal diseases in hematopoietic stem cell transplantation. Curr Opin Hematol. 2014;21:459-65.

28. Gao L, Zhang C, Gao L, Liu Y, Su Y, Wang S, et al. Favorable outcome of haploidentical hematopoietic stem cell transplantation in Philadelphia chromosome-positive acute lymphoblastic leukemia: a multicenter study in Southwest China. J Hematol Oncol. 2015:8:90.

29. Aguado JM, Vázquez L, Fernández-Ruiz M, Villaescusa T, Ruiz-Camps I, Barba $P$, et al. Serum galactomannan versus a combination of galactomannan and polymerase chain reaction-based Aspergillus DNA detection for early therapy of invasive aspergillosis in high-risk hematological patients: a randomized controlled trial. Clin Infect Dis. 2015;60:405-14.

30. White PL, Parr C, Thornton C, Barnes RA. Evaluation of real-time PCR, galactomannan enzyme-linked immunosorbent assay (ELISA), and a novel lateralflow device for diagnosis of invasive aspergillosis. J Clin Microbiol. 2013;51:1510-6.

31. Petrik M, Franssen GM, Haas $H$, Laverman $P$, Hörtnagl $C$, Schrettl $M$, et al. Preclinical evaluation of two 68Ga-siderophores as potential radiopharmaceuticals for Aspergillus fumigatus infection imaging. Eur J Nucl Med Mol Imaging. 2012;39:1175-83.

32. Johnson G, Nolan T, Bustin SA. Real-time quantitative $P C R$, pathogen detection and MIQE. Methods Mol Biol. 2013;943:1-16.

33. Marinach-Patrice C, Fekkar A, Atanasova R, Gomes J, Djamdjian L, Brossas JY, et al. Rapid species diagnosis for invasive candidiasis using mass spectrometry. PLOS ONE. 2010:5:e8862.

34. Johnson G, Ferrini A, Dolan SK, Nolan T, Agrawal S, Doyle S, et al. Biomarkers for invasive aspergillosis: the challenges continue. Biomark Med. 2014;8:429-51. 
35. Döring M, Eikemeier M, Cabanillas Stanchi KM, Hartmann U, Ebinger M, Schwarze CP, et al. Antifungal prophylaxis with posaconazole vs. fluconazole or itraconazole in pediatric patients with neutropenia. Eur J Clin Microbiol Infect Dis. 2015:34:1189-200.

36. Fleming S, Yannakou CK, Haeusler GM, Clark J, Grigg A, Heath CH, et al. Consensus guidelines for antifungal prophylaxis in haematological malignancy and haemopoietic stem cell transplantation, 2014. Intern Med J. 2014;44:1283-97.

37. Vehreschild MJ, von Bergwelt-Baildon M, Tran L, Shimabukuro-Vornhagen A, Wisplinghoff $\mathrm{H}$, Bangard C, et al. Feasibility and effectiveness of posaconazole prophylaxis in combination with micafungin bridging for patients undergoing allogeneic stem cell transplantation: a 6-yr analysis from the cologne cohort for neutropenic patients. Eur J Haematol. 2014;93: 400-6.

38. Tragiannidis A, Dokos $\mathrm{C}$, Lehrnbecher $\mathrm{T}$, Groll AH, Groll AH. Antifungal chemoprophylaxis in children and adolescents with haematological malignancies and following allogeneic haematopoietic stem cell transplantation. Drugs. 2012;72:685-704.

39. Wang JF, Xue Y, Zhu XB, Fan H. Efficacy and safety of echinocandins versus triazoles for the prophylaxis and treatment of fungal infections: a metaanalysis of RCTs. Eur J Clin Microbiol Infect Dis. 2015;34:651-9.

40. Takagi S, Araoka H, Uchida N, Uchida Y, Kaji D, Ota H, et al. A prospective feasibility study of primary prophylaxis against invasive fungal disease with voriconazole following umbilical cord blood transplantation with fludarabine-based conditioning. Int J Hematol. 2014;99:652-8.

41. Liu Q, Lin R, Sun J, Xiao Y, Nie D, Zhang Y, et al. Antifungal agents for secondary prophylaxis based on response to initial antifungal therapy in allogeneic hematopoietic stem cell transplant recipients with prior pulmonary aspergillosis. Biol Blood Marrow Transplant. 2014;20:1198-203.

42. Vehreschilda JJ, Sieniawski M, Reuterb S, Arenza D, Reichertd D, Maertense J, et al. Efficacy of caspofungin and itraconazole as secondary antifungal prophylaxis: analysis of data from a multinational case registry. Int J Antimicrobl Agents. 2009;34:446-50.

43. Bow EJ, Vanness DJ, Slavin M, Cordonnier C, Cornely OA, Marks DI, et al. Systematic review and mixed treatment comparison meta-analysis of randomized clinical trials of primary oral antifungal prophylaxis in allogeneic hematopoietic cell transplant recipients. BMC Infect Dis. 2015;15:128.

44. Sung AH, Marcella SW, Xie Y. An update to the cost-effectiveness of posaconazole vs fluconazole or itraconazole in the prevention of IFD among neutropenic patients in the United States. J Med Econ. 2015;18: 341-8.

45. Groll AH, Castagnola E, Cesaro S, Dalle JH, Engelhard D, Hope W, et al. Fourth European Conference on Infections in Leukaemia (ECIL-4): guidelines for diagnosis, prevention, and treatment of invasive fungal diseases in paediatric patients with cancer or allogeneic haemopoietic stem-cell transplantation. Lancet Oncol. 2014;15:e327-40.

46. van Hal SJ, Gilroy NM, Morrissey CO, Worth LJ, Szer J, Tam CS, et al. Survey of antifungal prophylaxis and fungal diagnostic tests employed in malignant haematology and haemopoietic stem cell transplantation (HSCT) in Australia and New Zealand. Intern Med J. 2014:44:1277-82.

47. Held J, Schmidt T, Thornton CR, Kotter E, Bertz H. Comparison of a nove Aspergillus lateral-flow device and the Platelia ${ }^{\oplus}$ galactomannan assay for the diagnosis of invasive aspergillosis following haematopoietic stem cell transplantation. Infection. 2013;41:1163-9.

48. Ji Y, Xu LP, Liu DH, Chen YH, Han W, Zhang XH, et al. Positive results of serum galactomannan assays and pulmonary computed tomography predict the higher response rate of empirical antifungal therapy in patients undergoing allogeneic hematopoietic stem cell transplantation. Biol Blood Marrow Transplant. 2011;17:759-64

49. Omer AK, Ziakas PD, Anagnostou T, Coughlin E, Kourkoumpetis T, McAfee $S L$, et al. Risk factors for invasive fungal disease after allogeneic hematopoietic stem cell transplantation: a single center experience. Biol Blood Marrow Transplant. 2013;19:190-6.

\section{Submit your next manuscript to BioMed Central and we will help you at every step:}

- We accept pre-submission inquiries

- Our selector tool helps you to find the most relevant journal

- We provide round the clock customer support

- Convenient online submission

- Thorough peer review

- Inclusion in PubMed and all major indexing services

- Maximum visibility for your research

Submit your manuscript at www.biomedcentral.com/submit
Biomed Central 